Research Article

\title{
Characteristics of Centrifugal Pumps Working in Direct or Reverse Mode: Focus on the Unsteady Radial Thrust
}

\author{
Anthony Couzinet, Laurent Gros, and Daniel Pierrat \\ Cetim, 74 route de la Jonelière, 44326 Nantes, France \\ Correspondence should be addressed to Anthony Couzinet; anthony.couzinet@cetim.fr
}

Received 25 February 2013; Revised 20 June 2013; Accepted 20 June 2013

Academic Editor: J-C. Han

Copyright ( 2013 Anthony Couzinet et al. This is an open access article distributed under the Creative Commons Attribution License, which permits unrestricted use, distribution, and reproduction in any medium, provided the original work is properly cited.

Experimental and numerical investigations have been carried out to study the behaviour of a centrifugal pump operating in direct mode or turbine mode. First of all, the complete characteristics (head, power, and efficiency) were measured experimentally using a specific test loop. The numerical data obtained from a CFD study performed with the ANSYS CFX software and based on steady state and unsteady approaches were compared to the experimental results. The representation in the 4 operating quadrants shows the various operating zones where the head is always positive. Then, the unsteady radial forces were analysed from transient computations. The results obtained for the pump operation are consistent with the literature and extended to the nonnormal operating conditions, namely, for very high flowrate values. The evolution of the radial load during turbine operation is presented for various partial flow operating points.

\section{Introduction}

The hydraulic performances of centrifugal pumps were widely studied experimentally over the last century, for normal operating modes close to the nominal operating point. For specific states of flow, the unsteady behaviour of the flow due to the rotor/stator interactions formed the subject of many studies. The experimental study of these unsteady phenomena requires complex and/or expensive experimental methods [1-3] such as the use of dynamic pressure sensors or strain gauges installed on the pump shaft, and, as a result, the numerical approach becomes a real alternative. As a matter of fact, CFD computations have been commonly used for approximately twenty years to predict the hydraulic performance of rotating machines. First of all, CFD computations made it possible to study and improve the design of blades. For that purpose, the use of periodical conditions became a means to reduce the size of the computational domain as well as the CPU time. Furthermore, it was possible to use a steady state approach to study operating points located close to the best efficiency point. Then, to study the rotor/stator interactions, the complete geometry of the pump needs to be integrated into the numerical model; it is also necessary to simulate these flow configurations in a transient manner $[1,4]$ in order to correctly predict all potential hydrodynamic instabilities. Nevertheless, although many projects focus on rotor/stator interactions, very few of them propose comparisons between numerical and experimental results. The only existing results concern the fluctuating pressure field at impeller outlet [5].

Even if a few studies focused on the abnormal operating conditions of centrifugal pumps, this subject has been much less studied, but it has become again a point of interest over the past 5 years. On the one hand, accident scenarios can be integrated into the test specifications, and therefore the performances of the pump operating in abnormal conditions have to be known. On the other hand, a growing number of small hydroelectric power stations (5 to $100 \mathrm{~kW} \mathrm{[6])} \mathrm{are}$ being developed due to their extremely attractive operating costs. However, the initial investment for the equipment is rather high. This is why the use of standard range centrifugal 
pumps operating in turbine mode has become a credible alternative to hydraulic turbines since their much lower cost and the wide variety of machines (in terms of operating points and dimensions) make it possible to significantly reduce equipment costs. Consequently, centrifugal pumps as turbines ("PAT") have become the main subject of an increasing number of studies, and the first work started approximately twenty years ago. The main objective of these studies concerns the analytical development of correlations whose purpose is to assess the hydraulic performance of the machine running in turbine mode, based on its pump operation characteristics. The use of CFD computations is rather new [7-11] for simulating the flow generated in the pump and for optimising the design of PATs. However, numerical results become all the more credible when they are accompanied by experimental results.

During this study, suitable experimental means and a sophisticated numerical model made it possible to study the complete characteristics of a centrifugal pump with a specific speed equal to 70 . It is possible to represent these characteristics on a speed versus flow diagram which materialises a representation in 4 quadrants (Knapp diagram [12]) as shown in Figure 1. These four quadrants correspond to four different operating modes. As a result, operation in pump mode is located in quadrant $Q_{1}$, operation in reverse pump mode is located in quadrant $Q_{2}$, operation in turbine mode is located in quadrant $Q_{4}$, and operation in reverse turbine mode is located in quadrant $Q_{3}$. We will particularly focus on the operating zones where $H$ is positive, which means the points located below the asymptotes $H=0$ in Figure 1.

Experimental data have been compared to the numerical results obtained from steady state and transient simulations. These results are very rich in terms of information: on the one hand, the overall characteristics in the 4 operating quadrants can be used to predict the behaviour of the machine during transient operations; on the other hand, local fluctuations in the flow can be predicted using the transient numerical simulations, as long as the numerical model used (in particular the turbulence model) is able to reproduce the development of the turbulence structures induced by the flow configurations. The choice of turbulence model will then be discussed through an analysis of the turbulence structures and the representative turbulence scales.

Furthermore, based on unsteady computations, the fluctuations of the radial forces which act on the impeller have been studied in normal operating mode and in turbine operating mode. In pump operation mode, the "radial force versus flow" curve has a conventional "V" shape. This curve is extended to the abnormal operating points (viz., when the head and torque become negative). This force has a privileged direction which is dependent on the operating point, while the fluctuations found for each pump operating point remain moderate. In turbine operation, the radial force is not stable, and its direction changes periodically over time depending on the operating point. This effect is demonstrated with the transient numerical simulations performed.

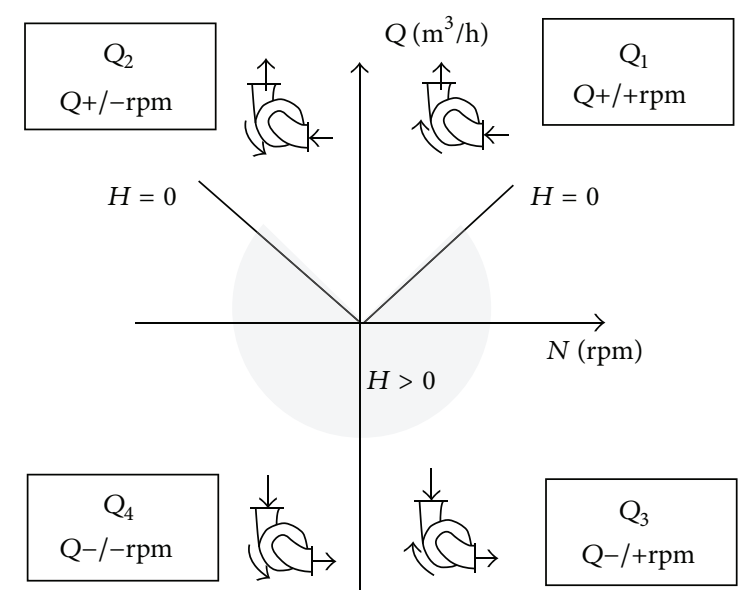

FIGURE 1: The four-quadrant representation: operating zones where $H>0$.

\section{Experimental Approach}

2.1. Description of the Test Loop and Design of the Centrifugal Pump. The centrifugal pump used in this study is comprised of a volute and a closed impeller with inlet and outlet diameters equal to $300 \mathrm{~mm}$ and $250 \mathrm{~mm}$, respectively. The characteristics at the best efficiency point are as follows: $N=$ $1,450 \mathrm{rpm}, Q_{n}=787.5 \mathrm{~m}^{3} / \mathrm{h}$, and $H_{n}=21 \mathrm{~m}$. The specific speed of the pump is $N_{s}=70$ (3,595 in US units). The pump was tested in a test loop dedicated to the measurements of the characteristics in the 4 quadrants. Following, the test rig is described.

(i) It is possible to set accurately the operating points thanks to supplying pumps which can ensure $5000 \mathrm{~m}^{3} / \mathrm{h}$.

(ii) A $90 \mathrm{~kW}$ direct current motor powered by a variable frequency controller.

(iii) Motorized discharge valves of the pumping station for the control of the flow rate.

(iv) An electromagnetic flow meter (accuracy 0.5\%) set as far as possible from the pump exit.

(v) Pressure transmitters (accuracy 0.3\%) located at the inlet and outlet sections and giving the average tip pressure on four pressure tapping.

(vi) A torque meter set between the pump and the motor.

\section{Results}

We mainly focused on quadrants $Q_{1}$ and $Q_{4}$ which correspond to operation in pump mode or turbine mode for normal operating conditions. The overall characteristics of the centrifugal pump in these two operating modes are illustrated in Figures 2 and 3. The head and the power are represented by the dimensionless variables $\psi=g H / N^{2} D^{2}$ 


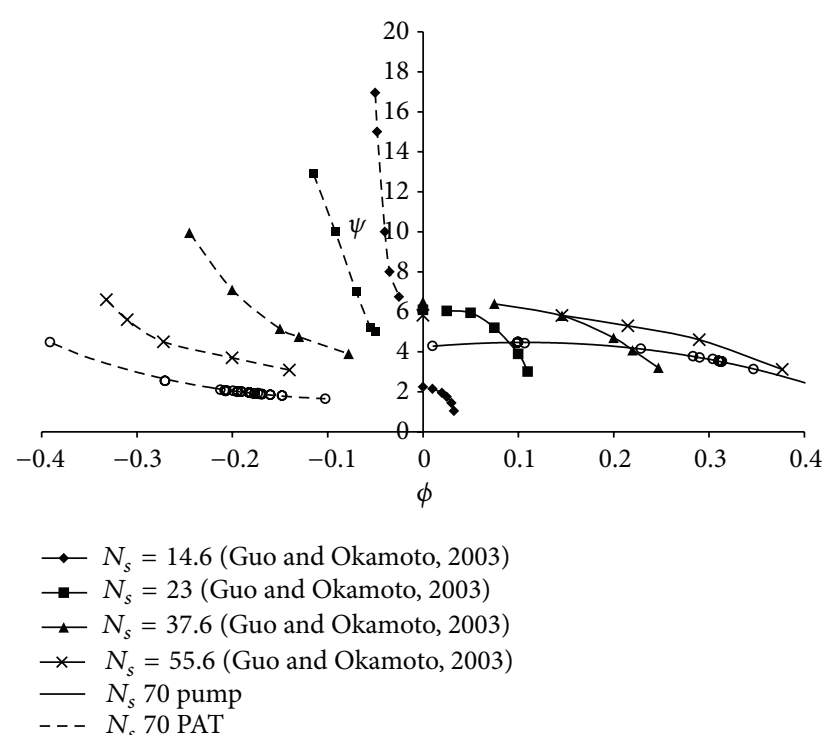

Figure 2: Dimensionless head curves of PAT in pump and turbine modes-comparison to Derakshan et al.

and $\pi=P / \rho N^{3} D^{5}$ versus the dimensionless flow rate $\phi=$ $\mathrm{Q} / N D^{3}$

The curves are consistent with the experimental results presented by Derakhshan et al. $[9,13,14]$ for volute centrifugal pumps with a specific speed varying from 15 to 60 . All these results are compared in the same figures. The positive flowrates correspond to the operating points in pump mode whereas the negative flowrates correspond to the operating points in turbine mode. This convention was adopted for all representations.

The efficiency curves are plotted in the same way in Figure 4. Whatever the specific speed considered, the efficiency values obtained in pump or turbine operation are similar. In fact, the PAT's efficiencies are quite good because the hydraulic design of this centrifugal pump is similar to the Francis turbines and the head losses for pump or turbine mode are similar.

Based on their test campaign, Derakhshan and Nourbakhsh [14] proposed several correlations which depended on the specific speed. The purpose of these correlations was to assess the hydraulic performance of the pump operating in turbine mode, based on the characteristics at the pump best efficiency point. So, the dimensionless BEP characteristics of the tested PAT are defined as following:

$$
h=\frac{H_{n, t}}{H_{n}} \quad q=\frac{Q_{n, t}}{Q_{n}} \quad p=\frac{P_{n, t}}{P_{n}} \quad \lambda=\frac{\eta_{n, t}}{\eta_{n}},
$$

where $H, Q, P$, and $\eta$ are the head, flow rate, power, and efficiency.

These correlations are illustrated in Figure 5 and compared with Derakshan's experimental results. The characteristics predicted from the correlations proposed for the centrifugal pump of this study are illustrated in this figure. This prediction method is based on the experimental results

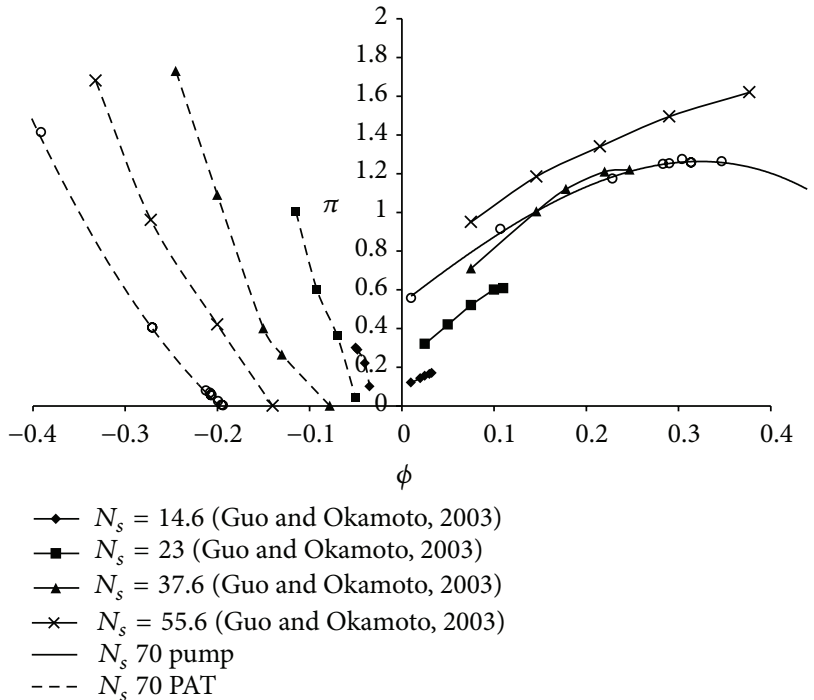

FIGURE 3: Dimensionless power curves of PAT in pump and turbine modes-comparison to Derakshan et al.

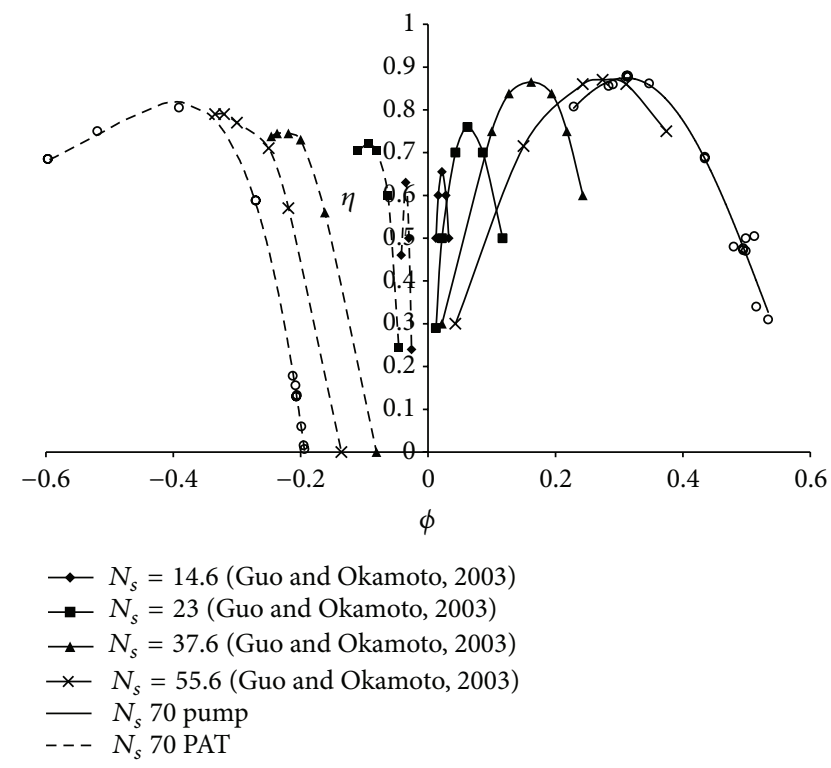

Figure 4: Dimensionless efficiency curves of PAT in pump and turbine modes-comparison to Derakshan et al.

obtained on four centrifugal pumps with similar geometry in which only the hydraulics of the impeller was modified to change the specific speed from 14.6 to 55.6 ; it seems to be unsuitable for centrifugal pumps with high specific speeds.

\section{Numerical Approach}

All numerical simulations were carried out with ANSYS CFX 11, and the computations were performed on workstations with the following technical characteristics: HP xw8600-8 


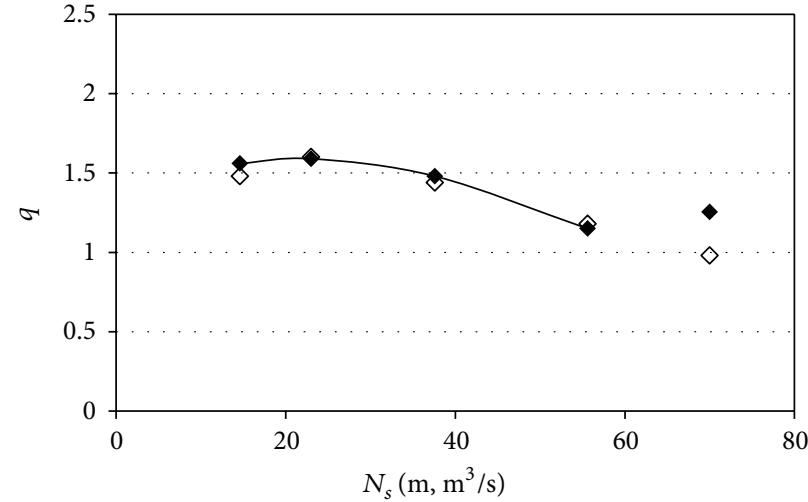

(a)

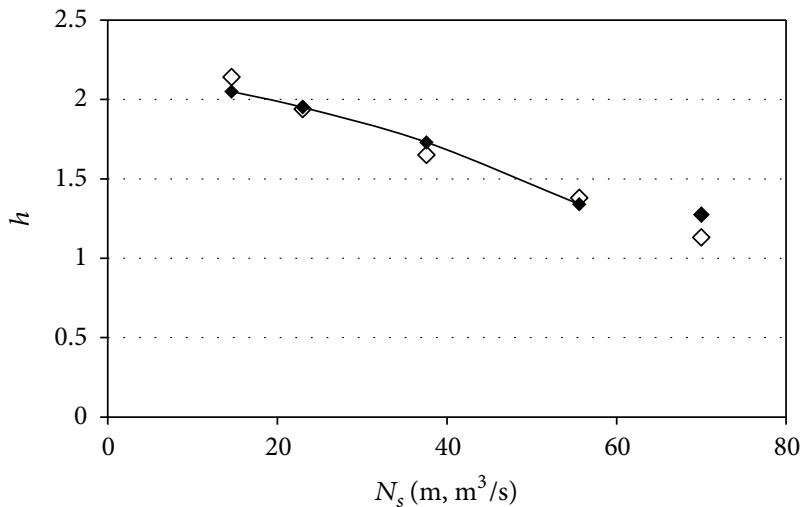

(b)

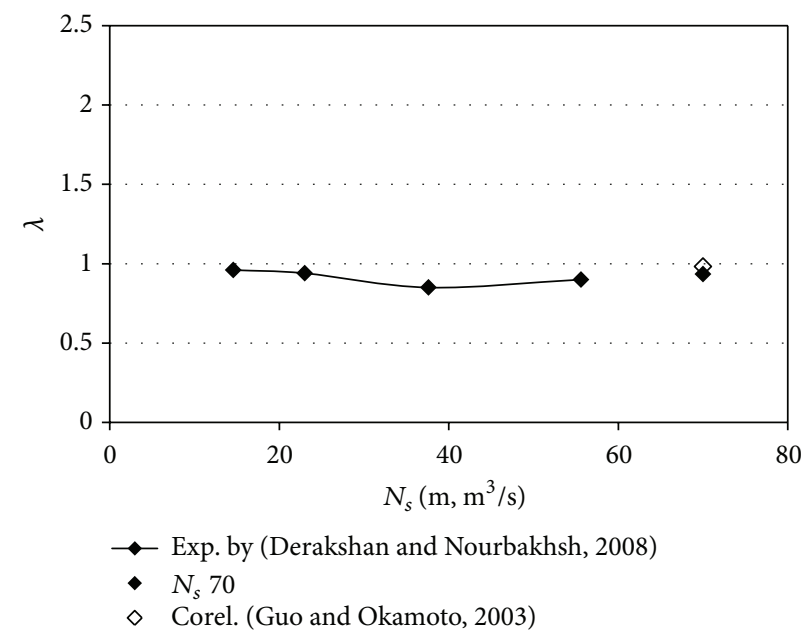

(c)

FIgUre 5: Dimensionless BEP characteristics of PATS—experiments by Derakhshan and Nourbakhsh [14] and tested pump.

Xeon processors (3.16 GHz), RAM $16 \mathrm{~Gb}$, Windows XP. The average number of processors is equal to 10 .

4.1. Computational Domain. The numerical model of the centrifugal pump is comprised of the volute, a six-blade impeller with balancing holes, and a leakage gap at the back of the impeller. So as to obtain the best possible comparison with the test campaign, all the geometrical details of the pump are taken into account as described in Figure 6. However, the surfaces of the various parts of the computational domain need to be cleaned to ensure optimum meshing quality.

The computational domain is broken down into three parts: the inlet duct, the rotating domain, and the volute. The positions of the interfaces between the rotating part and the stationary parts are defined in Figure 6. Each colour represents one of the three parts of the computational domain. The inlet and outlet of the domain are positioned, respectively, at $2 \mathrm{D}$ upstream and $3 \mathrm{D}$ downstream.

4.2. Hexahedral Structured Meshing. The meshing for the computational domain was constructed from a structured multiblock approach based on hexahedral elements. In comparison with nonstructured or hybrid approaches, this type of computation grid gives better meshing quality in the impeller while it simultaneously keeps a reasonable number of cells. Furthermore, considering the control-volume finiteelement method (CVFEM) formulation implemented in the ANSYS CFX computation code, the use of structured grids remains the best guarantee to obtain accurate numerical solutions.

The meshing is generated with the ICEM CFD software. The inlet duct, the impeller, and the volute are fully meshed, and the size of the generated grids reaches 450,000 , $4,900,000$, and 2,300,000 elements, respectively. A view of the meshing of the impeller is illustrated in Figure 7.

The meshing is refined in the vicinity of the walls in order to correctly obtain the peripheral velocity gradients and the friction effects: the average value of $y+$ is equal to 50; specific cell thickness progression laws in the meridian, hub-to-shroud, and blade-to-blade directions are applied to ensure good grid quality: near wall orthogonality is enforced, and the minimum angle observed in the domain corresponds to $23^{\circ}$ (less of $1 \%$ of the cells are smaller than $30^{\circ}$ ). 


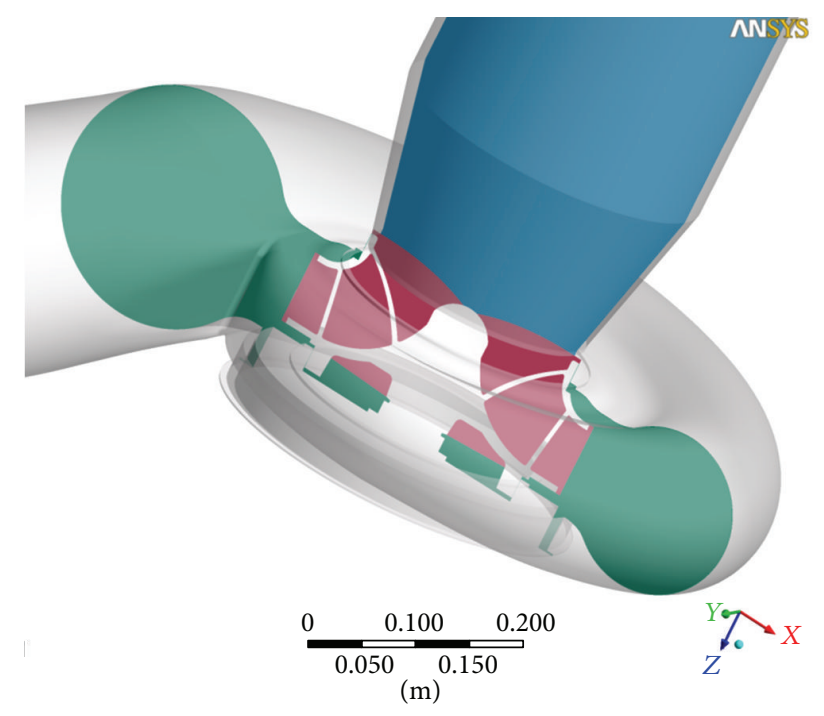

Figure 6: Cross-section of the computational domain.

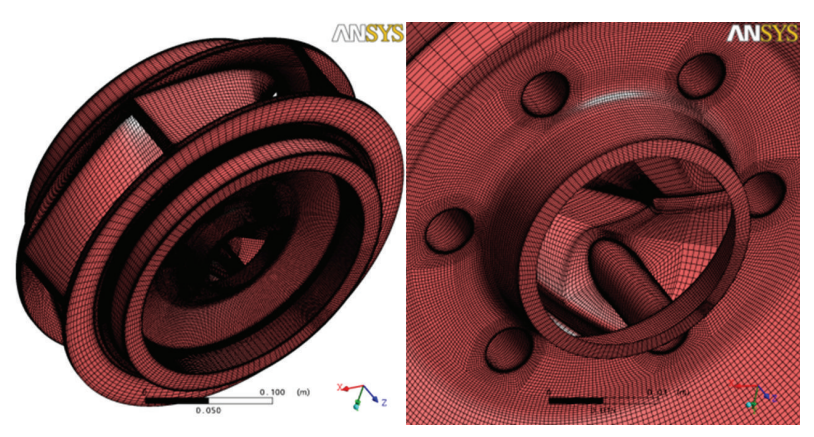

Figure 7: Mesh visualization of impeller.

4.3. Operating and Boundary Conditions. The physical characteristics of the fluid correspond to the characteristics of water: density is set to $1,000 \mathrm{~kg} / \mathrm{m}^{3}$, and dynamic viscosity is set to $0.001 \mathrm{~kg} \cdot \mathrm{m}^{-1} \cdot \mathrm{s}^{-1}$.

An even flowrate is imposed as an input for the computational field, while a constant static pressure is set for the output of the computational domain. We do not use any condition of periodicity to simulate the flow in the pump, as the flow in the volute is completely simulated.

For the steady state simulations, the interface between the stationary parts and the rotating part requires the use of a model which allows information to be transferred between both parts while integrating the Coriolis effects: we tested "stage" type approaches (azimuthal average of the velocity field) and "frozen rotor" type approaches (fixed rotor position). The computations performed in transient operation integrate the displacement of the meshing of the rotating part at each time iteration. (The time increment used is thus related to the angular displacement of the rotating part.) In actual practice, an angular increment of $1^{\circ}$ to $2^{\circ}$ is enough to accurately simulate this type of configuration.

The accuracy of the time and space discretisation schemes is of the second order. More precisely, the convective flows are assessed using the Barth and Jespersen method, which is similar to a TVD_MUSCL scheme [3, 15-17].

The turbulence is modelled from a two-equation approach based on the turbulent viscosity concept. The arguments related to the choice of the turbulence model (in particular SST or $k$-eps) are discussed in a previous document [18]. Considering the work previously carried out, only the results obtained in the $k$-eps model are presented in this paper.

\section{Overall Characteristics of the Centrifugal Pump}

5.1. Definition of the Reduced Variables. Based on the definitions proposed in Knapp's article and based on the laws of similarity of centrifugal pumps, it is possible to determine a set of reduced variables for the flowrate and the speed velocity as a function of the head of the pump:

$$
Q_{1 H}=\frac{Q}{D^{2} \sqrt{H}} \quad N_{1 H}=\frac{N D}{\sqrt{H}} .
$$

In order to extend these reduced variables for the abnormal operating conditions, the head and torque values can be substituted by their absolute value. Moreover the values at the nominal operating point in pump mode allow this set of reduced variables to be made dimensionless which allows us to write the following relations:

$$
Q_{1 H}^{*}=\frac{Q}{Q_{n}} \frac{D_{n}^{2}}{D^{2}} \frac{\sqrt{H_{n}}}{\sqrt{|H|}}, \quad N_{1 H}^{*}=\frac{N}{N_{n}} \frac{D}{D_{n}} \frac{\sqrt{H_{n}}}{\sqrt{|H|}} .
$$

All the numerical and experimental results will be represented considering these two variables, by means of the curve $Q_{1 H}^{*}=f\left(N_{1 H}^{*}\right)$.

5.2. Steady State Computations. As a first approach, steady state computations were carried out in the 4 operating quadrants in order to simulate the behaviour of the centrifugal pump in all operating conditions, thus making it possible to determine the position of the $H=0$ asymptotes. As regards quadrant $Q_{1}$, the position of the asymptote is correctly predicted, and the crossing of the head/flowrate curve at the zero head point is located at approximately $1.8 Q_{n}$. Anyway, the prediction of the asymptote in quadrant $Q_{2}$, namely, in reverse pump operation, is unrealistic. The head value is still negative, even for very low flowrates. This malfunction is visible in Figure 9, where the grey curve represents the evolution of the reduced variables.

5.3. Unsteady Approach-Transient Computations. Although the steady state approach seems to be sufficient to correctly describe the behaviour of the flow in the entire quadrants $Q_{1}$ and $Q_{3}$, a few transient computations were performed. As a matter of fact, five points were simulated for these operating modes in order to demonstrate the capability of the steady state approach to provide reliable solutions in terms of head, torque, and force. On the contrary, it was demonstrated that 


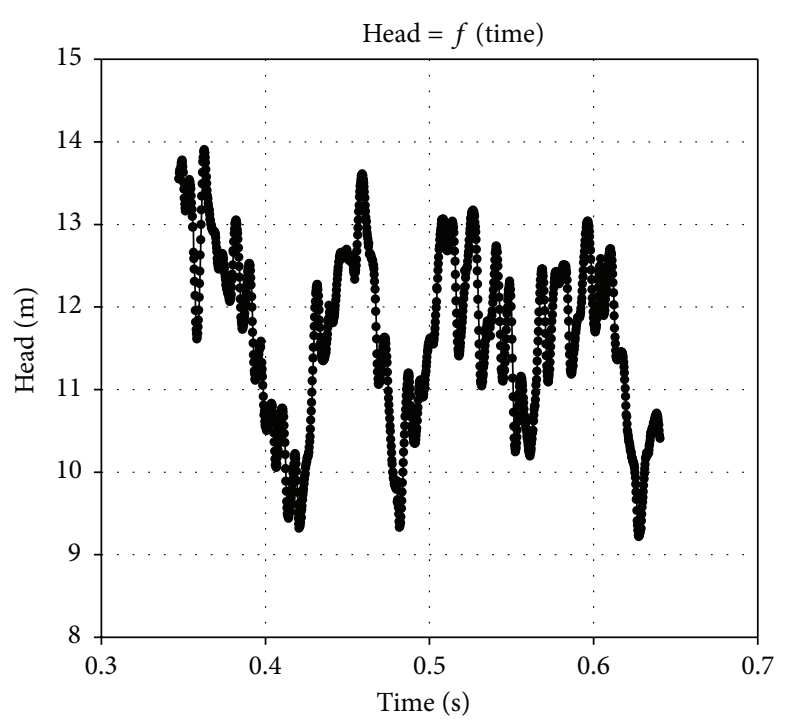

(a)

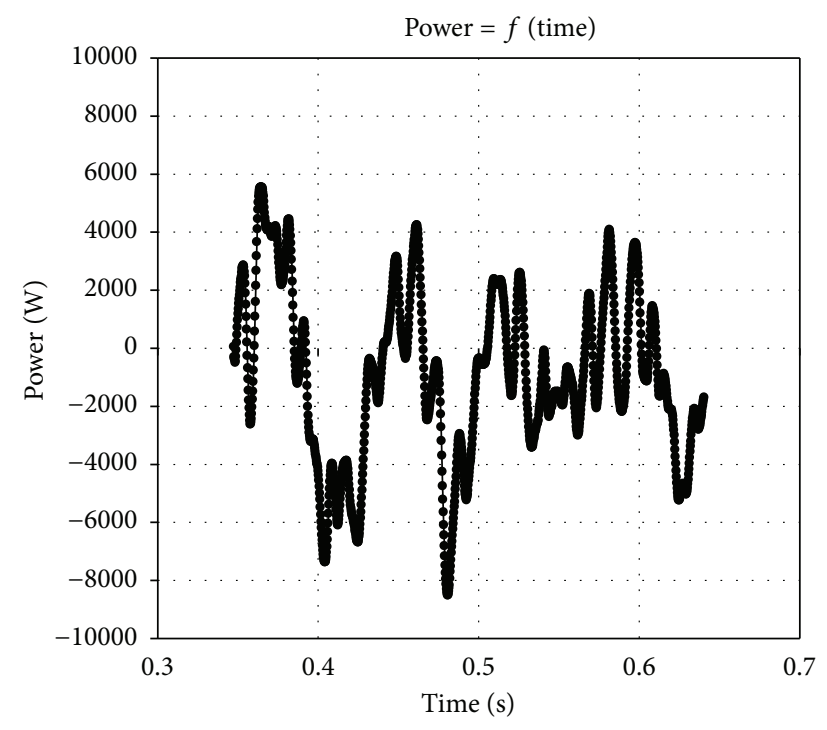

(b)

FIGURE 8: Time evolutions of head and power for an unsteady computation-operating point: $-\operatorname{rpm} / Q=-0.6 Q_{n}$.

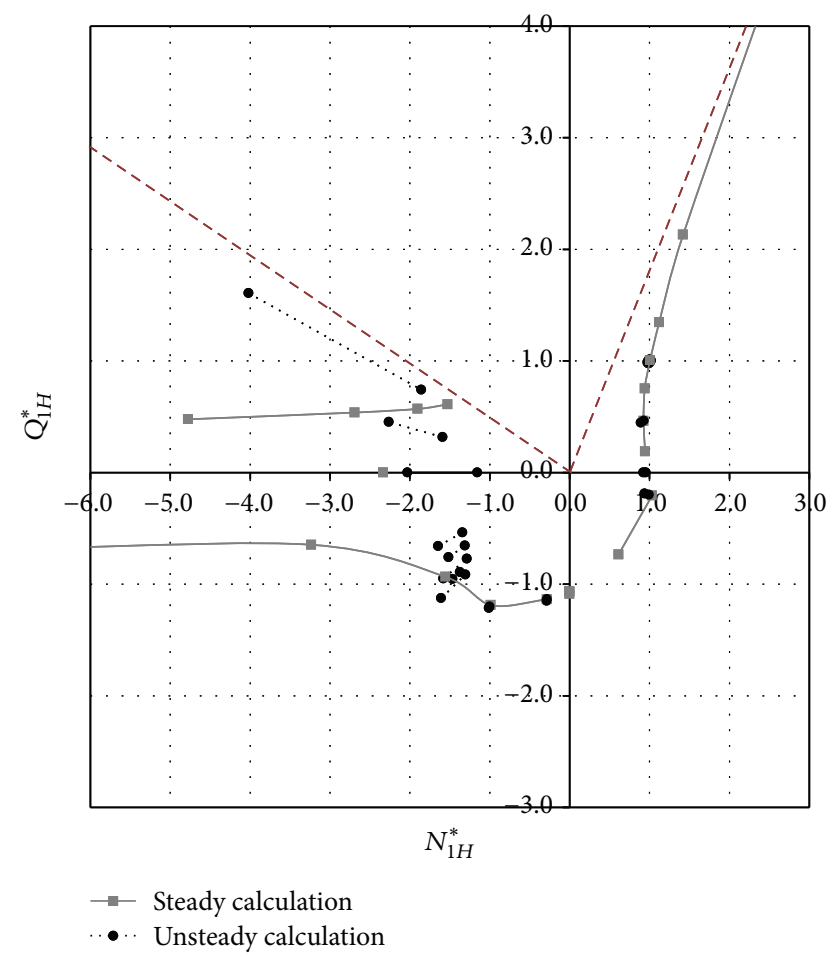

FIGURE 9: Numerical results obtained with steady state calculations and unsteady approaches.

the steady state approach gives unrealistic solutions for most of the operating points of quadrants $Q_{2}$ and $Q_{4}$. This is why a specific effort was implemented to simulate a wide number of operating points for these states of flow, which represented in total 6 and 7 operating points in quadrants $Q_{2}$ and $Q_{4}$.
The choice of these various operating points allowed us to correctly describe the characteristic curve $Q_{1 H}^{*}=f\left(N_{1 H}^{*}\right)$ and determine the position of the asymptote $H=0$ in quadrant $Q_{2}$.

All transient computations were performed with a time step corresponding to an impeller angular increment of $2^{\circ}$. This value has been optimised so as to obtain an acceptable compromise between computation time and result accuracy. The gap between the impeller and the volute is rather significant, which does not require high-frequency sampling. However, several impeller rotations (up to eight rotations) may be necessary to correctly acquire instabilities such as "rotating stalls" which occur in certain operating conditions. In the end, the levels of RMS residuals of the time resolution are excellent for all the cases, and they reach a value close to $10^{-6}$.

The time evolutions of the overall characteristics are followed-up for all computational cases. If the head and power fluctuations are not significant for quadrants $Q_{1}$ and $Q_{3}$, thus substantiating that the steady state is sufficient for these cases, these fluctuations are much more significant for quadrants $Q_{2}$ and $Q_{4}$. This occurs in particular for the operating points where the flowrate is moderate, as shown in Figure 8, where the time evolutions of head and power are represented for the operating point $-0.6 Q_{n} /-\mathrm{rpm}$.

The minimum and maximum values of the fluctuations are then used to compute the minimum and maximum values of the corresponding reduced variables. The interval between these two extreme values is then represented on the curve $Q_{1 H}^{*}=f\left(N_{1 H}^{*}\right)$ of Figure 9 for each operating point (black circle symbols) in comparison to the results obtained with the steady state approach. As we supposed, the results obtained in transient operation in quadrants $Q_{1}$ and $Q_{3}$ are similar to the steady state results, while 


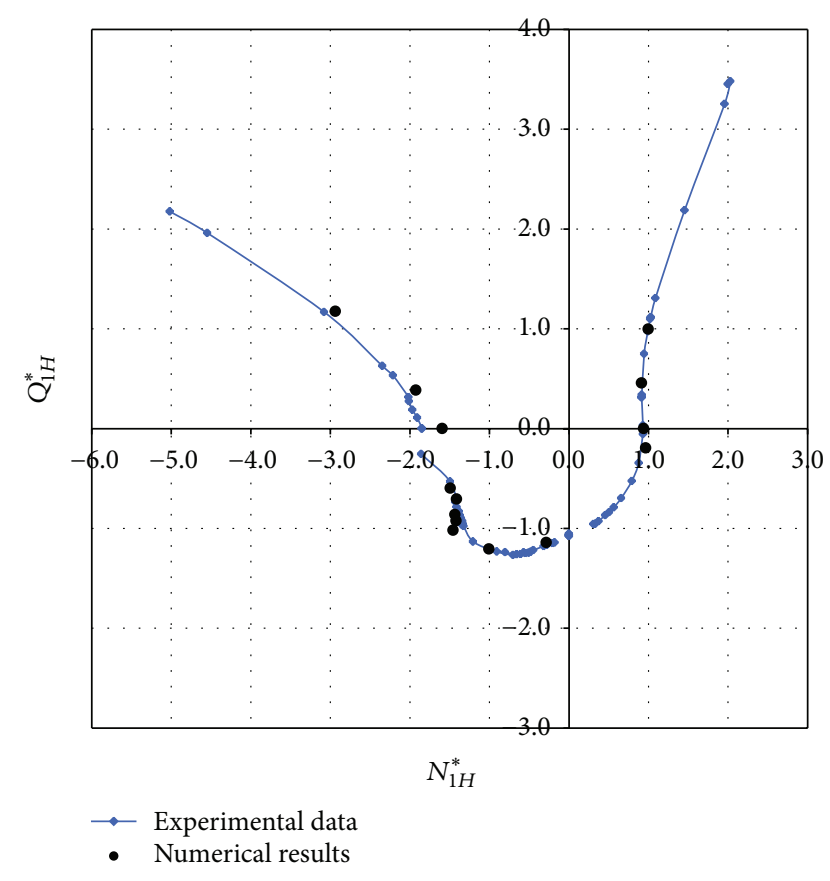

FIGURE 10: Comparisons between experimental data and numerical results obtained from unsteady approach.

the values corresponding to the other two quadrants give more realistic results showing an asymptote $H=0$ in quadrant $Q_{2}$ with very significant fluctuations along this asymptote.

Based on the fluctuating values of the reduced variables, an average curve is plotted to describe the numerical behaviour of the curve $Q_{1 H}^{*}=f\left(N_{1 H}^{*}\right)$ in the 4 operating quadrants, and it is compared to the experimental results in Figure 10. We can see a very good correlation between the experimental measurements and the numerical results. The maximum difference occurs for the points located in the vicinity of the $N_{1 H}^{*}=0$ axis: whether in the quadrant $Q_{1}$ or $Q_{2}$ that is to say on either side of the axis, these operating points correspond to high flowrate values. It is worth reminding that the development of cavitation within the pump is not taken into account in the numerical model. For these operating points, the head drop generated by the appearance of cavitation may explain these differences. However, it seems rather unlikely that the use of Rayliegh-Plesset models to simulate the cavitating flow will improve the prediction of the overall variables because the pressure drop due to the appearance of cavitation is significantly underestimated with this type of model $[19,20]$.

\section{Radial Thrust and Forces Fluctuations on the Impeller}

At the nominal operating point, the centrifugal pump impellers are (in practice) correctly balanced, and the radial thrust becomes negligible. Further from the nominal operating point, the flow becomes unsuitable, and local stalls may appear which is likely to generate a radial thrust whose amplitude and direction mainly depend on the operating conditions. This static force has been widely studied by a great number of authors $[1,2,4,21]$. The "radial thrust versus flowrate curve" has a typical "V" form. This conventional form is obtained numerically: the minimum radial thrust is obtained for the best efficiency point, and it increases for the points with partial flowrate or with excess flow (Figure 13). The dimensionless factor $k_{r}$ is then defined by the relation below:

$$
k_{r}=\frac{F_{r}}{\rho g H b_{2} d_{2}} .
$$

The components of this factor are illustrated in Figure 11 for the three following operating points: $0.5 Q_{n}, Q_{n}$, and $2.2 Q_{n}$. For each operating point, we compared the solutions in steady state and unsteady modes. The force directions are correctly predicted with the steady state approach since the fluctuations of direction are low, but the amplitude may be widely underestimated in particular for underflow operating points.

The direction of the radial thrust with respect to the volute varies with the operating point, and it is dependent on the type of machine, as Gülich explains [21]. This direction goes towards the outlet for underflow conditions and in the opposite direction for excess flowrate conditions. Although this result is widely known for excess flowrates up to $1.5 Q_{n}$ (normal pump operating conditions), the simulated operating point at $2.2 Q_{n}$ corresponds to an abnormal operating point where the head and torque are negative, which allows us to extent these results (direction and amplitude of the radial force) to abnormal operating points.

To select the suitable equipment which will be installed in hydroelectric power stations, it is crucial to predict the hydraulic performance of centrifugal pumps in PAT operation. As we mentioned in the introduction and the first part of this paper, there are correlations which should make it possible to assess the performance in turbine mode, based on pump operation data. However, the prediction of the hydraulic load is also extremely important in order to guarantee the mechanical integrity of the pump in this new operating mode. As a matter of fact, except for turbine pumps, the pumps which operate in turbine mode (pumps as turbine, PAT) are not specifically designed for these operating ranges. This is why we have studied the radial thrust for several operating points in PAT: $0 Q_{n}, 0.4 Q_{n}$, $0.6 Q_{n}, 1.2 Q_{n}$, and $4 Q_{n}$. In particular, we analysed the load fluctuations which may appear depending on the operating conditions. For underflow operating points, it appears that the fluctuating components of the radial thrust are generated by a low-frequency hydraulic imbalance. Therefore, the numerical representation of this physical phenomenon requires several simulated impeller rotations. For example, 8 impeller rotations were simulated for the operating point $-0.6 Q_{n}$.

The temporal evolution of the radial load is illustrated in Figure 12 for the various simulated flowrates. The components of the $k_{r}$ factor are plotted for each iteration. At 


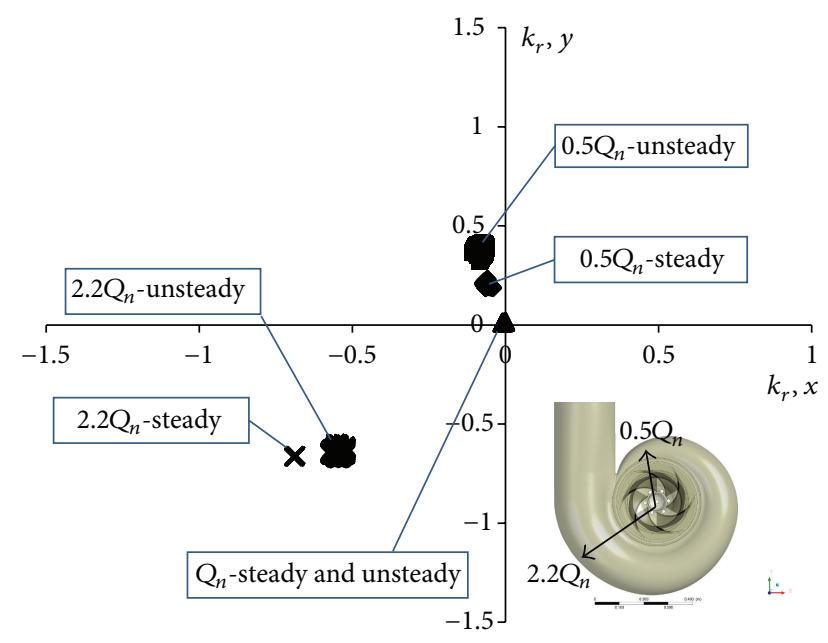

FIGURE 11: Components of radial thrust in pump mode $0.5 Q_{n}, Q_{n}$, and $2.2 Q_{n}$.

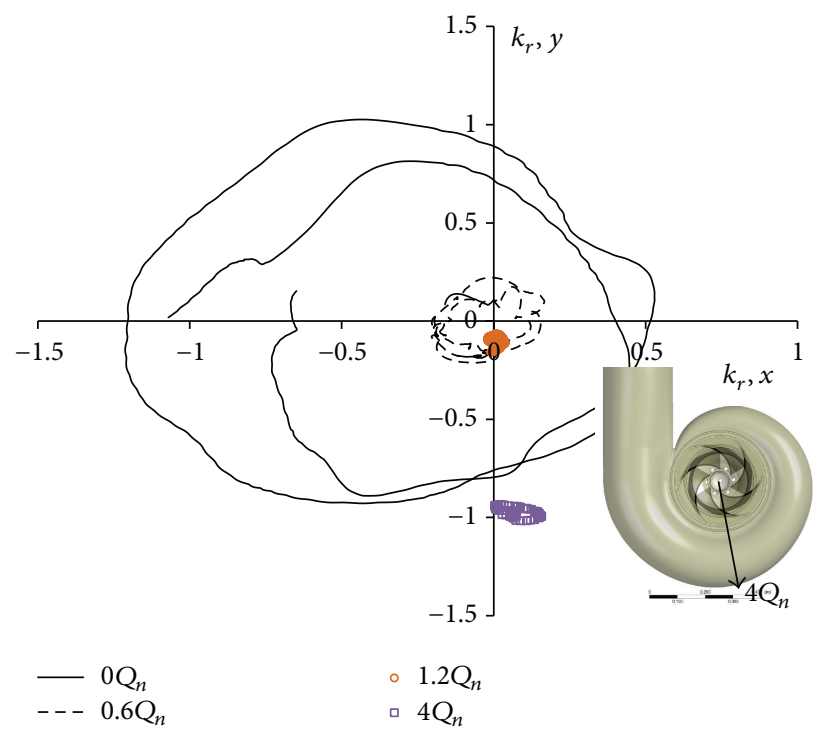

FIgURE 12: Components of radial thrust in PAT mode.

the same time, the average amplitude of the radial force is illustrated in Figure 13 as a function of the flowrate. The behaviour of the radial thrust in PAT operation is similar to that obtained in pump operation. The minimum radial force is obtained for the best efficiency point in turbine mode $\left(1.2 Q_{n}\right)$. The PAT configuration exhibits radial force levels similar to those obtained in pump operation. For the excess flowrate point, the fluctuating part of the radial force is very low in comparison to the average value, and its direction remains constant and opposite to the turbine inlet (Figure 12). For the operating points with underflow and in particular for operating points close to runway, the radial force becomes a rotating force centred on the impeller. Finally, the average amplitude of the radial force increases when the flowrate decreases.

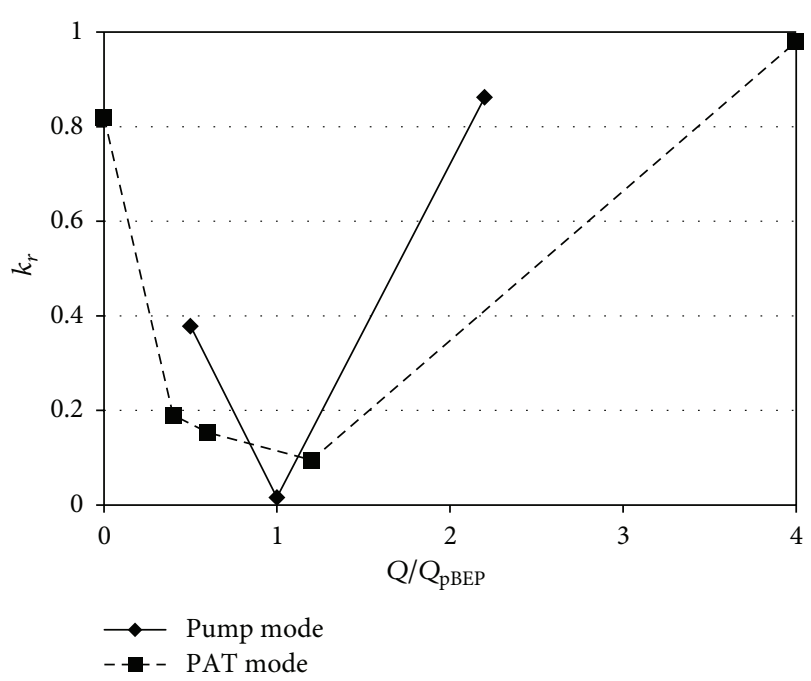

FIGURE 13: Averaged amplitude of the unsteady radial force.

The instantaneous pressure field is illustrated in Figure 14 for six different instants, and the corresponding radial thrust is represented by a superimposed vector. The computation code gives access to the radial force in the rotating frame, but it can be recomputed in the stationary frame if the angular position of the impeller is known, which makes it possible to represent this force over time. The amplitude and direction of the radial thrust change with the relative position of the impeller, and this evolution is pseudoperiodic. This physical phenomenon is due to the rotating stalls which occur in a few channels of the impeller and move with the rotation of the impeller. They are induced due to complex interactions [22-24] which appear in underflow conditions and lead to interaction between the pumping and turbining effects. These phenomena also exist for turbine pumps, and they have been studied by several authors $[22,25,26]$.

Then, the time spectrum of the radial thrust is calculated. Two main frequencies are then identified in Figure 15. Then $f_{n}$ is the rotating frequency of the pump, while the low frequency corresponding to the displacement of the rotating stall is equal to $0.6 f_{n}$. This result is known for hydraulic turbines: these characteristic frequencies are located between $0.5 f_{n}$ and $0.8 f_{n}$, depending on the geometry of the turbine [26-28]. Furthermore, the frequency of passage of one blade at each impeller rotation $\left(6 f_{n}\right)$ which is detected by the head fluctuation spectrum is not clearly identified on this spectrum.

\section{Conclusions}

The performance of a centrifugal pump with a specific speed of 70 in pump and turbine operating modes was measured on a dedicated test loop. These measurements are compared to the results obtained by Derakshan concerning four centrifugal pumps with volute from $N_{s} 14$ to 56 . The results obtained 

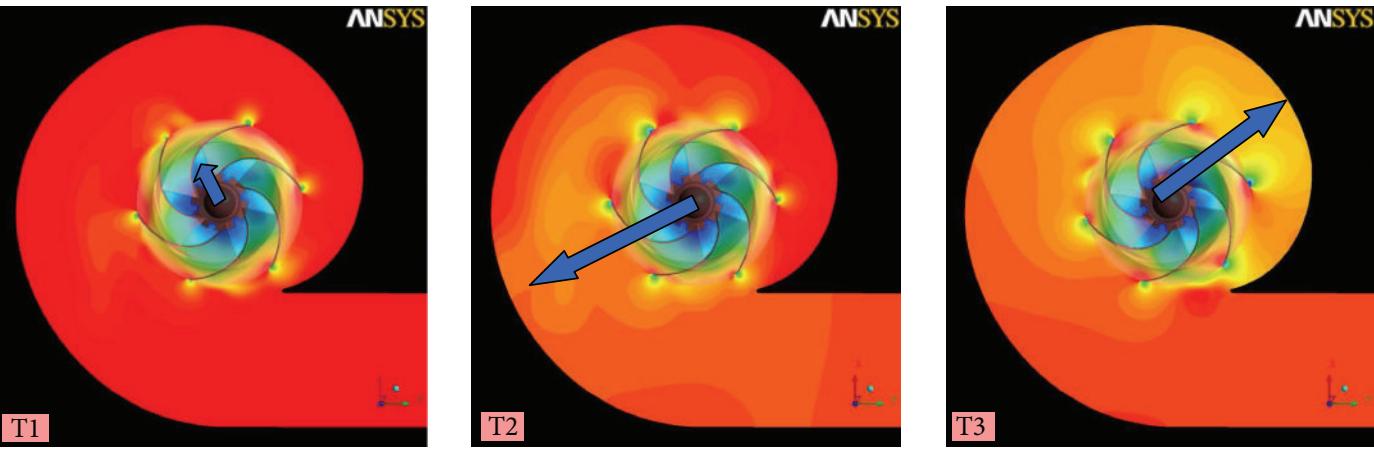

Pressure

(contour 1)

140000

130588

121176

111765

102353

92941

83529 三

74118

ॠ 64706

45882

36471

27059

17647

8235

$-1176$

$-10588$

$-20000$

(a)
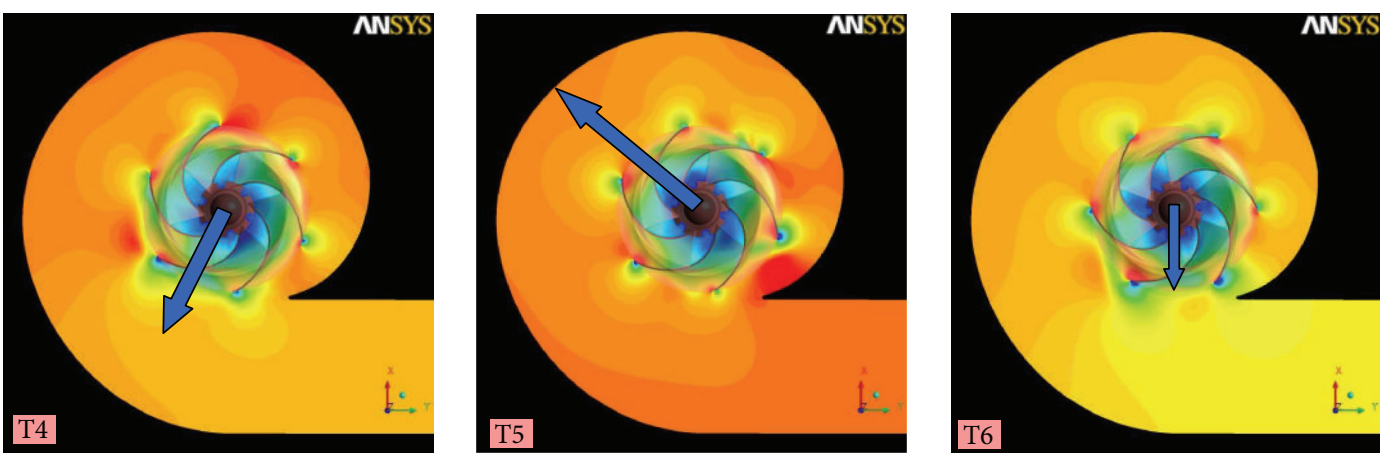

Pressure

(contour 1)

140000

130588

121176

111765

102353

92941 三

83529 三

74118

ส 64706

55294

45882

36471

27059

17647

8235

$-1176$

$-10588$

$-20000=$

(b)

FIGURE 14: Instantaneous pressure field and radial trust evolution for the following operating point: $-\mathrm{rpm} / \mathrm{Q}=-0.6 Q_{n}$.

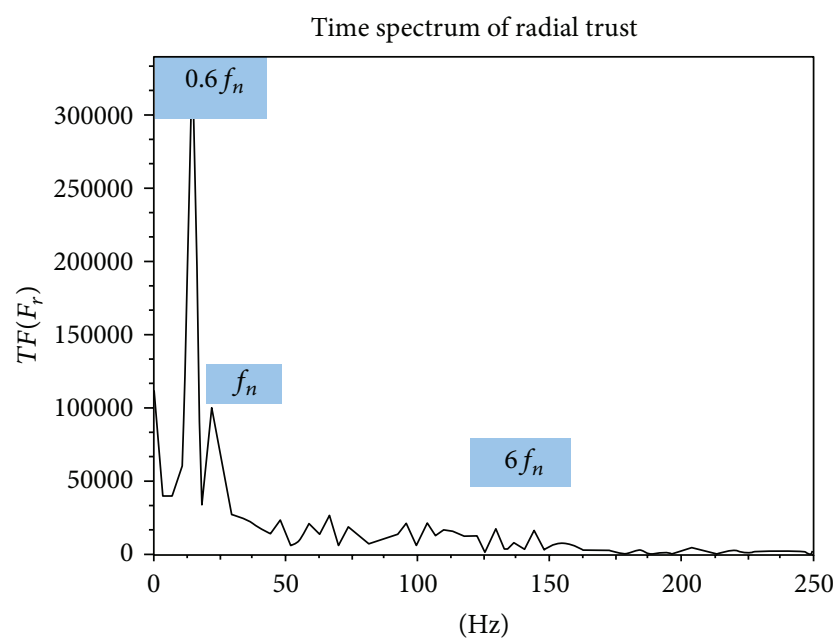

FIGURE 15: Spectrum of the fluctuations of radial thrust.

show that the correlation proposed by Derakshan seems to be unsuitable for high values of $N_{s}$.
The overall characteristics stemming from experimental results and from numerical solutions are very similar. In turbine operation, the comparisons remain good if an unsteady approach is used. The hydraulic load is then studied in order to verify the complete validity of the numerical approach. In pump operation, the conventional " $\mathrm{V}$ " shape of the "radial thrust versus flowrate" curve is extended to the abnormal operating points (excessive flowrates such as $H<0$ and $C<$ 0 ). In turbine operation, the average radial thrust has a similar $\mathrm{V}$ shape where the minimum value is obtained for the best efficiency point in turbine mode. Furthermore, for underflow operating points close to the runway point, the radial force is a rotating force whose rotating frequency corresponds to 0.6 times the rotation frequency of the pump. This result agrees with the results from the literature concerning turbine pump operation.

The good comparisons with the experimental data show that the URANS modelling is able to solve the large energy scales maintained by a forcing mechanism due to the rotation of the impeller. As regards applications in rotating machinery, the traditional URANS approaches are a credible alternative in comparison to advanced turbulence models which require a much higher CPU time to be allocated. 


\section{Nomenclature}

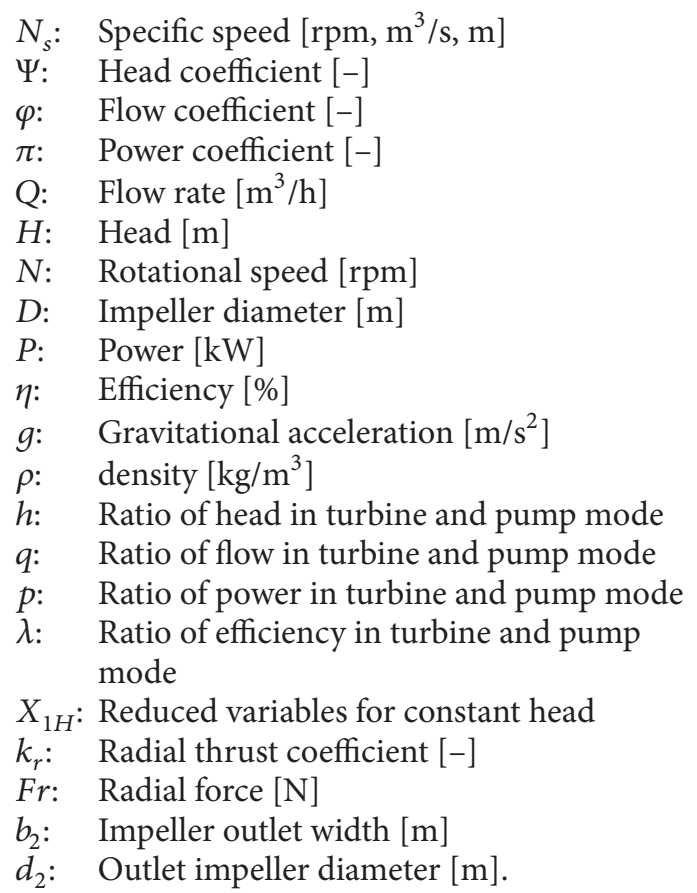

\section{Subscripts}

$n$ : Nominal point

$t$ : Turbine mode.

\section{Acknowledgments}

The authors would like to thank the French pump manufacturers and the members of the CETIM working group on the operating conditions of centrifugal pumps in the 4 quadrants who have proposed and supported this work.

\section{References}

[1] J. F. Combes, A. Boyer, L. Gros, D. Pierrat, G. Pintrand, and P. Chantrel, "Experimental and numerical investigations of the radial thrust in a centrifugal pump," in Proceedings of the 12th International Symposium on Transport Phenomena and Dynamics of Rotating Machinery, pp. 1-7, Honolulu, Hawaii, USA, 2008, ISROMAC12-2008-20044.

[2] S. Guo and H. Okamoto, "An experimental study on the fluid forces induced by rotor-stator interaction in a centrifugal pump," International Journal of Rotating Machinery, vol. 9, no. 2, pp. 135-144, 2003.

[3] F. R. Menter, "A comparison of some recent eddy-viscosity turbulence models," Journal of Fluids Engineering, vol. 118, no. 3, pp. 514-519, 1996.

[4] M. Asuaje, F. Bakir, S. Kouidri, F. Kenyery, and R. Rey, "Numerical modelization of the flow in centrifugal pump: volute influence in velocity and pressure fields," International Journal of Rotating Machinery, vol. 2005, no. 3, pp. 244-255, 2005.

[5] J. Parrondo-Gayo, J. Fernández-Francos, J. González-Pérez, and L. Fernández-Arango, "An experimental study on the unsteady pressure distribution around the impeller outlet of a centrifugal pump," in Proceedings of ASME Fluids Engineering Division Summer Meeting, 2000, ASME-FEDSM-00-11302.

[6] T. Agarwal, "Review of pump as turbine (PAT) for microhydropower," International Journal of Emerging Technology and Advanced Engineering, vol. 2, no. 11, pp. 163-168, 2012.

[7] R. Bario, J. Fernandez, J. Parrondo, and E. Blanco, "Performance prediction of a centrifugal pump working in direct and reverse mode using computational fluid dynamics," in Proceedings of the International Conference on Renewable Energies and Power Quality, Granada, Spain, 2010.

[8] S. Rawal and J. T. Kshirsagar, "Numerical simulation on a pump operating in a turbine mode," in Proceedings of the 23th International Pump Users Symposium, 2007.

[9] S. Derakhshan and A. Nourbakhsh, "Theoretical, numerical and experimental investigation of centrifugal pumps in reverse operation," Experimental Thermal and Fluid Science, vol. 32, no. 8, pp. 1620-1627, 2008.

[10] S. R. Natanasabapathi and J. T. Kshirsagar, "Pump as turbinean experience with CFX-5.6," Corporate Research and Eng. Division, Kirloskar Bros. Ltd.; 2004, http://www.ansys.com/ staticassets/ANSYS/staticassets/resourcelibrary/confpaper/ 2004-Int-ANSYS-Conf-127.pdf.

[11] H. Nautiyal, V. Varun, and A. Kumar, "Reverse running pumps analytical, experimental and computational study: a review," Renewable and Sustainable Energy Reviews, vol. 14, no. 7, pp. 2059-2067, 2010.

[12] R. T. Knapp, "Complete characteristics of centrifugal pumps and their use in prediction of transient behaviour," Transactions of the American Society of Mechanical Engineers, vol. 59, pp. 683689, 1937.

[13] S. Derakhshan, B. Mohammadi, and A. Nourbakhsh, "Efficiency improvement of centrifugal reverse pumps," Journal of Fluids Engineering, vol. 131, no. 2, Article ID 021103, 9 pages, 2009.

[14] S. Derakhshan and A. Nourbakhsh, "Experimental study of characteristic curves of centrifugal pumps working as turbines in different specific speeds," Experimental Thermal and Fluid Science, vol. 32, no. 3, pp. 800-807, 2008.

[15] D. Pierrat, L. Gros, and G. Pintrand, Modélisation des Écoulements Incompressibles, Approche Couplée-déCouplée, Les Ouvrages du Cetim.

[16] F. R. Menter, "Zonal two equation k-w turbulence models for aerodynamic flows," in Proceedings of the 24th Fluid Dynamics Conference, Orlando, Fla, USA, July1993, AIAA paper 93-2906.

[17] M. S. Darwish and F. Moukalled, "TVD schemes for unstructured grids," International Journal of Heat and Mass Transfer, vol. 46, no. 4, pp. 599-611, 2003.

[18] L. Gros, A. Couzinet, D. Pierrat, and L. Landry, "Complete pump characteristics and 4-quadrants diagram investigated by experimental and numerical approaches," in Proceedings of the ASME Conference, Hamamatsu, Japan, 2011, AJK2011_06067.

[19] D. Pierrat, L. Gros, G. Pintrand, B. Le Fur, and Ph. Gyomlai, "Experimental and numerical investigations of leading of leading edge cavitation in a Helico-Centrifugal Pump," in Proceedings of the 12th International Symposium on Transport Phenomena and Dynamics of Rotating Machinery, Honolulu, Hawaii, USA, February 2008, ISROMAC12-2008-20074.

[20] D. Pierrat, L. Gros, A. Couzinet, G. Pintrand, and Ph. Gyomlai, "On the leading edge cavitation in a helico-centrifugal pump: experimental and numerical investigations," in Proceedings of the 3rd IAHR International Meeting of the WorkGroup on 
Cavitation and Dynamic Problems in Hydraulic Machinery and Systems, Brno, Czech Republic, October 2009.

[21] J. F. Gülich, Centrifugal Pumps, Springer, New York, NY, USA, 2nd edition, 2004.

[22] V. Hasmatuchi, "Hydrodynamics of a pump-turbine at offdesign operating conditions: numerical simulations," in Proceedings of ASME-JSME-KSME Joint Fluids Engineering Conference (AJK2011-FED '11), Hamamatsu, Japan, July 2011.

[23] T. Staubli, F. Senn, and M. Sallaberger, "Instability of pumpturbines during start-up in the turbine mode," in Hydro, Ljubljana, Slovénie, 2008.

[24] Q. Liang and M. Keller, Behaviour of Pump Turbines Operating at Speed no Load Conditions in Turbine Mode, Hydro Vision, Charlotte, NC, USA, 2010.

[25] Q. Liang, M. Keller, and N. Lederegr, "Rotor-stator interaction during no-load operation of pump-turbines," in Hydro, Lyon, France, 2009, paper no. 7.8.

[26] J. Vesely, L. Pulpitel, and P. Troubil, "Model research of rotating stall on pump-turbines," in Hydro, Porto Carras, Greece, September 2006, paper no. 3.5.

[27] C. Widmer, T. Staubli, and N. Ledergerber, Unstable PumpTurbine Characteristics and Their Interaction with Hydraulic Sytems, Hydro Vision, Charlotte, NC, USA, 2010.

[28] L. Wang, J. Yin, L. Jiao, D. Wu, and D. Qin, "Numerical investigation on the "s" characteristics of a reduced pump turbine model," Science China Technological Sciences, vol. 54, no. 5, pp. 1259-1266, 2011. 

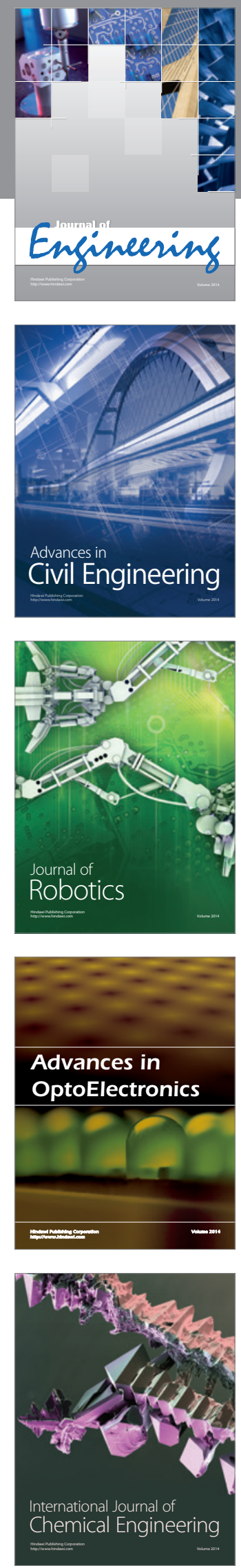

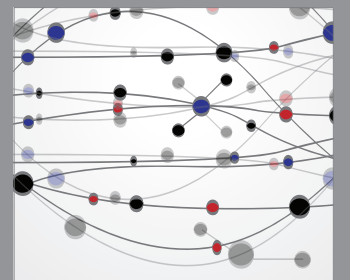

The Scientific World Journal
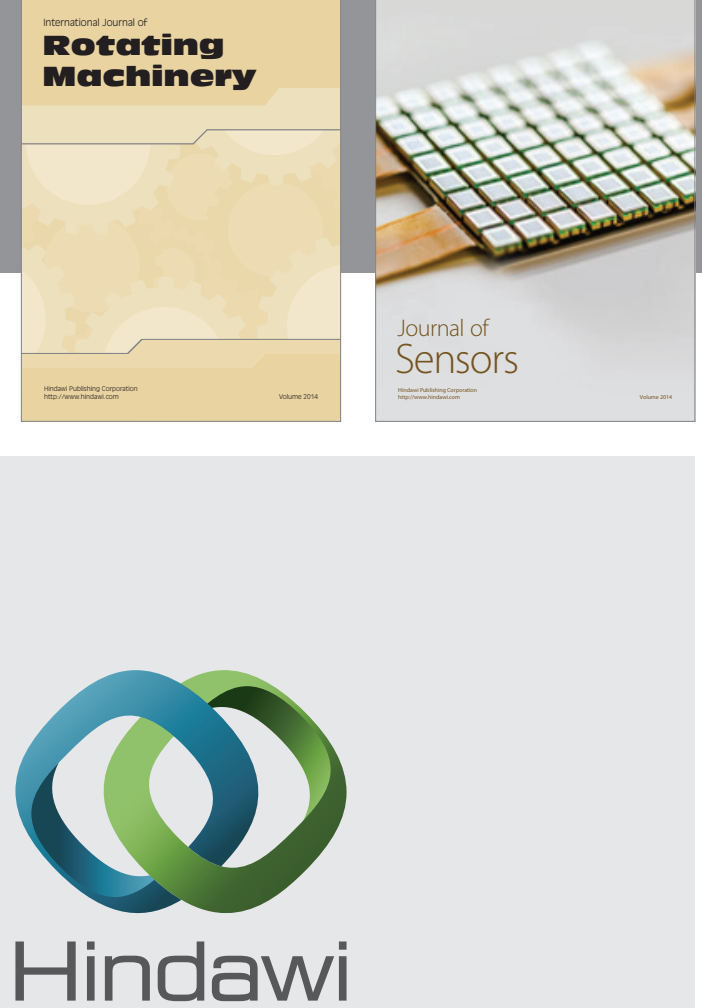

Submit your manuscripts at http://www.hindawi.com
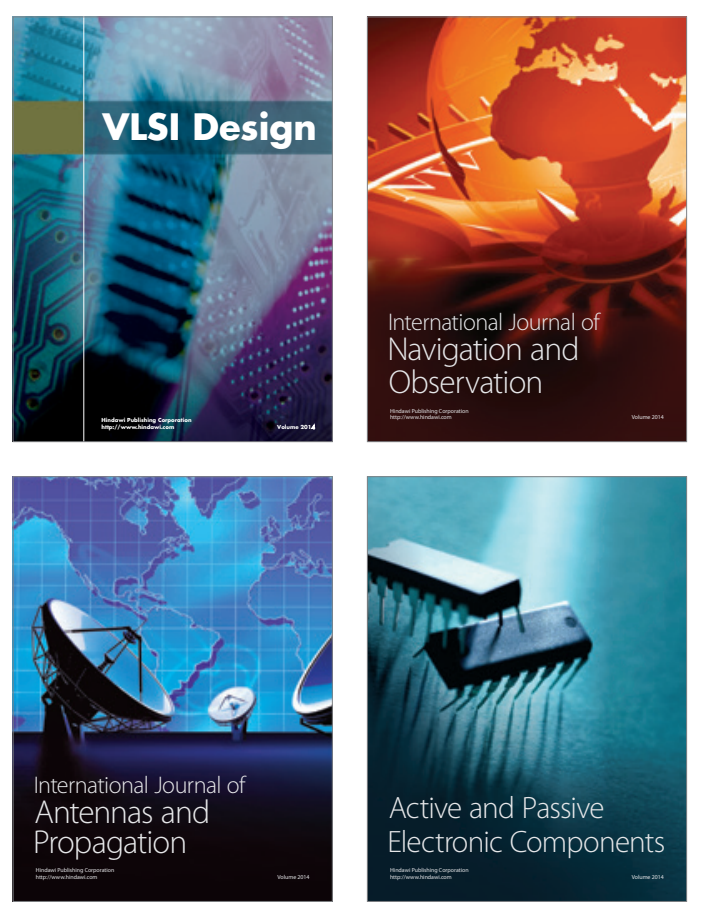
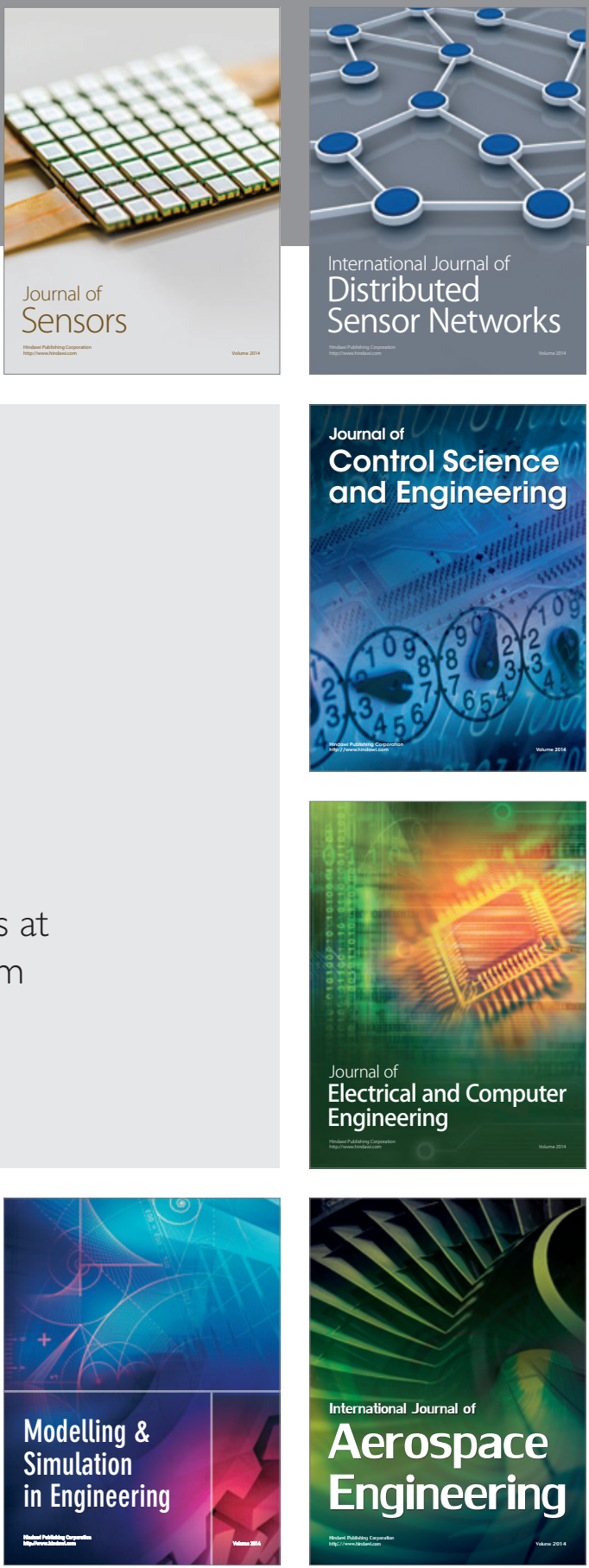

Journal of

Control Science

and Engineering
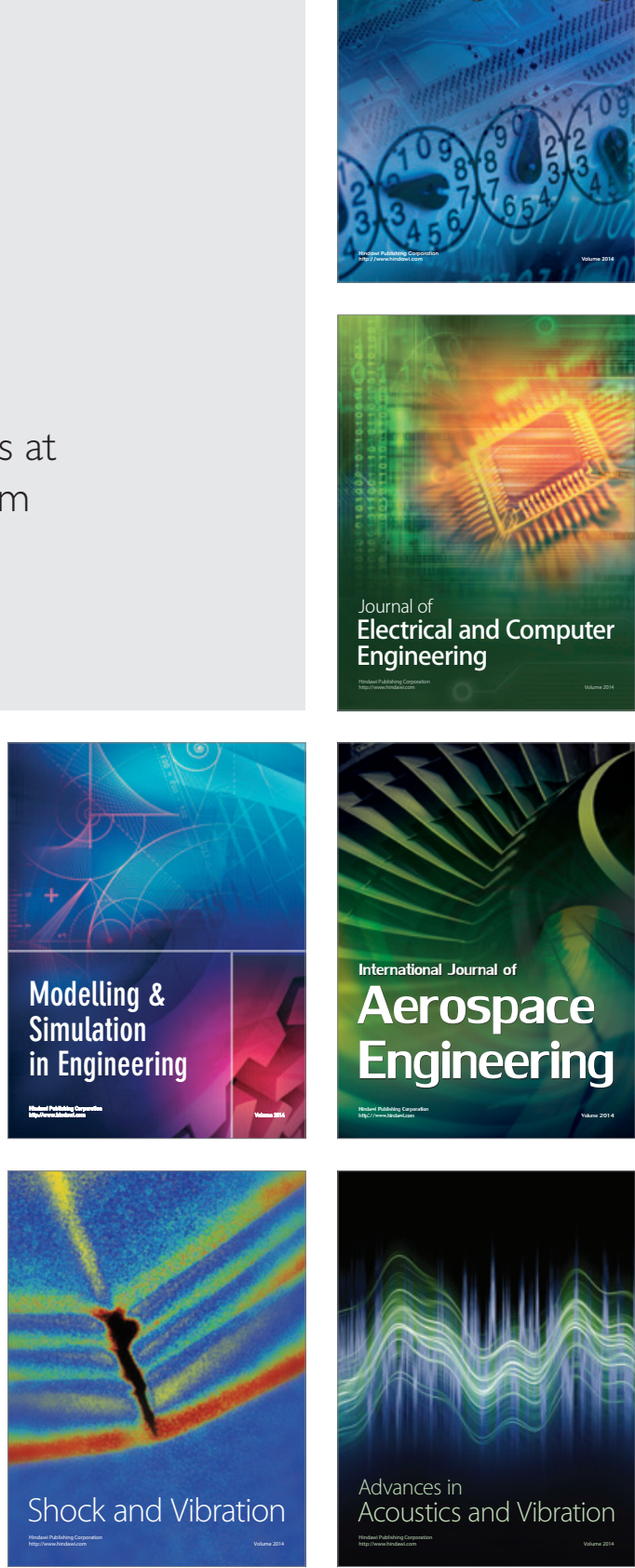